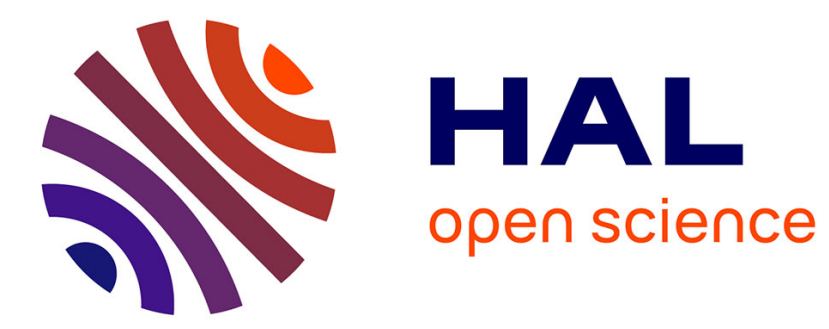

\title{
In Search of the Roots of Formal Computation
}

\author{
Jan Von Plato
}

\section{To cite this version:}

Jan Von Plato. In Search of the Roots of Formal Computation. 3rd International Conference on History and Philosophy of Computing (HaPoC), Oct 2015, Pisa, Italy. pp.300-320, 10.1007/978-3319-47286-7_21. hal-01615300

\section{HAL Id: hal-01615300 \\ https://hal.inria.fr/hal-01615300}

Submitted on 12 Oct 2017

HAL is a multi-disciplinary open access archive for the deposit and dissemination of scientific research documents, whether they are published or not. The documents may come from teaching and research institutions in France or abroad, or from public or private research centers.
L'archive ouverte pluridisciplinaire HAL, est destinée au dépôt et à la diffusion de documents scientifiques de niveau recherche, publiés ou non, émanant des établissements d'enseignement et de recherche français ou étrangers, des laboratoires publics ou privés. 


\title{
In search of the roots of formal computation
}

\author{
Jan von Plato \\ Department of Philosophy, University of Helsinki - jan.vonplato@ helsinki.fi
}

\begin{abstract}
In a culmination of a long development, it was seen clearly in the early 1930s that steps of formal computation are also steps of formal deduction as defined by recursion equations and other similar principles of arithmetic. Followers of Kant's doctrine of the synthetic a priori in arithmetic missed by a hair's breadth the proper recursive definition of addition that appeared instead first in a book of Hermann Grassmann of 1861. A line can be followed from it to Hankel, Schröder, Dedekind, Peano, and Skolem, the last mentioned marking the birth of recursive arithmetic, a discipline firmly anchored in the foundations of mathematics by the presentation Paul Bernays made of it in his monument, the Grundlagen der Mathematik of 1934.
\end{abstract}

\section{Introduction}

The basic laws of arithmetic have been an object of study at least since the times of Leibniz who thought he could prove them from suitable principles such as: "Those that can be mutually substituted salva veritate are equal." Formal arithmetic proper begins with the high school teacher Hermann Grassmann in 1861, otherwise known as the one who invented linear algebra and the vector calculus. He set out to prove some of the basic properties of arithmetic operations, and found that to prove the commutativity of addition, $a+b=b+a$, one has to prove first its associativity, $a+(b+c)=(a+b)+c$. The inductive proof for $a+b=b+a$ just doesn't go through if you don't do it in this order. Inductive proofs are rendered possible because Grassmann had found the correct definitions of the arithmetic operations. The source of these definitions seems to be in the development of combinatorics that provided recursively defined algorithms for the manipulation of formal expressio! ns.

The deductive part of arithmetic was developed in the work of Peano, Russell, and others, until in the 1920s a clear picture of elementary arithmetic as represented formally within predicate logic became standard. Predicate logic, i.e., the logic of the connectives and quantifiers, provided the deductive machinery by which elementary arithmetic could be defined as a theory within a formalized language, with the precisely defined questions of consistency, completeness, and decidability of the axiomatization to be answered.

Next to the deductive aspects of elementary arithmetic, there is the computational part that became gradually subsumed under deduction, as in the following succinct formulation of Bernays (in Hilbert and Bernays 1934, p. 288):

The formalization of the intuitive procedure of a recursive definition through the recursion scheme depends on obtaining, for a recursively introduced function sign ..., a derivable equation ... with the help of the recursion equations and the axioms of equality.

The way to a formal account of computation makes for a long story that begins with Leibniz and, perhaps surprisingly, Kant and especially the Kantian disciple Johann Schultz:

\section{First steps in formal arithmetic}

Properties of arithmetic operations were postulated axiomatically in earlier literature, as in the book Anfangsgründe der reinen Mathesis by Johann Schultz (Basics of pure mathesis, 1790). There the commutativity and associativity of addition appear as axioms (p. 41): 
1. The quantity of a sum is the same, should we add to the first given quantity the second or to the second the first, i.e., once and for all $a+b=b+a$, e.g., $5+3=3+5$.

2. The quantity of a sum is the same, should we add to the first given quantity another either once as a whole or each of its parts one after the other, i.e., once and for all $c+(a+b)=$ $(c+a)+b=c+a+b$.

These axioms were in Schultz' mind formulations of principles found in Kant's philosophy of arithmetic, as explained in his two-volume Prüfung der Kantischen Critik der reinen Vernunft (Examination of the Kantian critique of pure reason, 1789 and 1792) that went hand in hand with the mathematics of the Anfangsgründe of 1790.

Gottfried Martin studied these developments in his dissertation, published in 1938, in which he writes that the idea of axioms in arithmetic started to erode with Schultz. He refers to the question posed by Schultz (p. 57):

How do I know, then, that this willful procedure [of changing the order of a sum] that lies in no way in the concept of addition itself, provokes no difference in the sum $7+5$ ?

This passage is from the Prüfung (vol. 1, p. 220). Schultz gives there a proof of the equation $7+5=$ 12 - perhaps the only formula in Kant's Kritik, a "smoking gun" of sorts in later writers. Kant's most detailed formulation is the following (p. B16):

I take the number 7 to begin with and, using for the concept 5 the fingers of my hand as an intuitive help, I put the units that I earlier took together to make the number 5, now in that image of mine one by one to the number 7, and thus I see the number 12 emerge.

Schultz in turn gives first the axioms of commutativity and associativity of addition, then states that they are "indispensable for arithmetic" (p. 219), for without them, one would get only that $7+5=7+(4+1)=7+(1+1+1+1+1)$. With commutativity and associativity, one gets instead (p. 220):

Instead of all of 5, or its units taken together and added at once to 7, I must take them instead successively one by one, and instead of $7+(4+1)$ first set $7+(1+4)$, and in place of it $(7+1)+4$, so then I get thanks to the concept of the number 8 , namely that it is $7+1$, first $8+4$ i.e. $8+(3+1)$. Instead of this I have to set again $8+(1+3)$, and $(8+1)+3$ in place of it, so I get $9+3$, i.e., $9+(2+1)$. Setting for this $9+(1+2)$, and therefore $(9+1)+2$, gives $10+2$, i.e., $10+(1+1)$, and setting for this $(10+1)+1$ gives finally $11+1$, i.e. 12 . Every arithmetician knows that this is the only way through which we can come to the insight that the theorem is correct.

Here is a clear sign of awareness of the recursive definition of sum. Schultz missed by a hair's breadth the correct recursion equation, as can be seen by the comparison:

$$
\begin{aligned}
& \text { Schultz 1790: } 7+5 \stackrel{\text { def }}{=} 7+(4+1) \stackrel{\text { comm }}{=} 7+(1+4) \stackrel{\text { assoc }}{=}(7+1)+4 \\
& \text { Grassmann 1861: } 7+5 \stackrel{\text { def }}{=} 7+(4+1) \stackrel{\text { def }}{=}(7+4)+1
\end{aligned}
$$

He thus missed the inductive proofs of commutativity and associativity, and could not do better than claim that any attempted proof of the commutativity of addition would be circular (p. 221). In the Anfangsgründe, Schultz had declared the commutativity of addition to be "immediately evident" (p. 42).

Martin suggests that there is an intimation of deductive dependences in one of Schultz' discourses, namely, that the commutativity of product follows from the special case of $1 \times n=n \times 1$ and the distributive law. There is a grain of truth to this, as we shall see in a while.

Schultz' proof of $n \times r=r \times n$ is as follows: First the case $1 \times n=n \times 1$ is proved, and then come three lines with a first step on each line, Corollary 6, from the distributive law as in $n \times 2=n \times(1+1)=n \times 1+n \times 1$, and similarly for the last step, Corollary 4 . The "by proof" clause (p. dem.) in between refers always to the case of one less on a previous line (p. 64): 
Lemma 9. A product of two integers is the same, should one multiply the first factor by the second, or the second by the first, i.e., $n \times r=r \times n$. For since $n=1$ times $n(\S 36$. Cor. 3$)=n$ times 1 (\$36. Cor. 2$)$; then $n \times 1=1 \times n$.

$$
\begin{aligned}
& n \times 2=n \times 1+n \times 1 \text { (Cor. 6) }=1 \times n+1 \times n \text { (p. dem.) } \\
& =2 \times n \text { (Cor. } 4) \\
& n \times 3=n \times 2+n \times 1 \text { (Cor. } 6)=2 \times n+1 \times n \text { (p. dem.) } \\
& =3 \times n(\text { Cor. } 4) \\
& n \times 4=n \times 3+n \times 1 \text { (Cor. 6) }=3 \times n+1 \times n \text { (p. dem.) } \\
& =4 \times n \text { (Cor. } 4) \text { etc. }
\end{aligned}
$$

So we have in general $n \times r=r \times n$.

Martin (p. 61) calls this "an inference from $n$ to $n+1$," but it certainly is not the kind of induction in which we have a base case, here $r=1$, and a step case from an assumed value $r$ to $r+1$. There is instead a clear pattern indicated by the "etc" and the repetitive layout by which the proof of commutativity can be continued to any given number $r$. Let us single out the pattern by some little formal notation:

The expression $n \times r$ can be considered, for each value of $n$, a function of $r$ in the old-fashioned sense of an expression with a free variable $r$ for which values can be substituted as arguments of the function, denoted $f(r)$. Similarly, the expression $r \times n$ can be considered, for each value of $n$, a function of $r$, denoted $g(r)$. Schultz' first line of proof gives the result:

$$
f(1)=g(1)
$$

His general case, even if not written down with a variable, gives:

$$
\begin{aligned}
& f(r+1)=f(r)+f(1) \\
& g(r+1)=g(r)+g(1)
\end{aligned}
$$

Now we see the hidden inductive step:

$$
\text { If } f(r)=g(r) \text {, then } f(r+1)=f(r)+f(1)=g(r)+g(1)=g(r+1) \text {. }
$$

The base value for $r=1$ and the recursion clause from $r+1$ to $r$ in Schultz comes from his two corollaries, 2 and 3 in $\S 36$ and 6 in $\S 42, n=1 \times n=n \times 1$ and $n \times(r+1)=n \times r+n \times 1$. These are what we today take as the recursion equations for product; a concrete case of what I stated in the introduction, namely that inductive proofs showed the way to corresponding recursive definitions.

There is even a simpler way to look at Schultz' proof: We have two expressions $n \times r$ and $r \times n$. They agree in value for $r=1$ and when $r$ grows by one, each grows by the value $f(1)=g(1)$. Therefore $f(r)$ and $g(r)$ have the same value for all $r$.

The first one to have formulated Schultz' proof method explicitly, with no knowledge of this specific case of course, seems to be Paul Bernays in a talk he gave on 21 February 1928 in Göttingen, titled Die Rekursion als Grundlage der Zahlentheorie (Recursion as a foundation of number theory). Bernays, in a letter of 1940, mentions "the possibility of taking instead of the complete induction the rule of equalizing recursive terms satisfying the same recursive equations" (cf. von Plato 2014). An observation in much simpler words but to the same effect was made by Ludwig Wittgenstein some years after Bernays' talk, as reported in Waismann (1936, p. 99):

The induction proof can also be thought of as a direction for the formation of proofs of individual numerical equations, as the general terms of a series of proofs. Indeed, the induction proof could very well be written in the form of a series of equations, with individual numbers, as a part of a series with an "etc.," and it would thereby lose none of its power.

Seven years after the Anfangsgründe, Schultz produced an abridged version with the title Kurzer Lehrbegriff der Mathematik (Short course in mathematics) that has the theorem of commutativity of multiplication with an identical wording, and the proof (p. 36): 
For let one pose that the theorem be true for whatever multiplier $m$, namely let $n \times m=m \times n$; then $n \times(m+1)=n \times m+n \times 1$ (Cor. 5) $=m \times n+1 \times n(\$ 21$, Cor. 3. $)=(m+1) \times n($ Cor. 3 .), so the theorem is in this case true also for the successive multiplier $(m+1)$. The theorem is true for the multiplier 1 , because $n \times 1=1 \times n(\$ 21$, Cor. 3.) so also for the multiplier 2 , consequently also for the multiplier 3 etc, thereby for each multiplier $r$ whatever; i.e., in general $n \times r=r \times n$.

What made Schultz change the proof into an explicit induction? I have found no such proof in the Anfangsgründe, but it does have at least two proofs that use another variant of induction, remarkably both of them of the form of an indirect existence proof. Theorem 17 is (p. 84):

$\S 58$. Each composite finite number $m$ has a prime number as a divisor.

Proof. Since $m$ has a divisor $n(\S 57)$; we have $m=n+n+\ldots$ ( $\$ 53$. Cor. 1$)$. Let then $n$ be composite; then it again has a divisor $r$ and we have again $n=r+r+\ldots$, consequently $m=r+r+\ldots+r+r+\ldots$. Were $r$ again composite; then $r$ would again have a divisor $u$, and we would have $r=u+u+\ldots$, consequently $m=u+u+\ldots+u+u+\ldots+u+u+\ldots$. Then, had $m$ no prime number as a divisor, then each new divisor would have each time again a divisor without end, consequently $m$ would be a sum of infinitely many whole numbers, whereby it would be infinitely great $(\S 15)$. Because this is against the assumption, it must have a prime number as a divisor.

The theorem is followed by another by which "every composite finite number is a product of prime numbers throughout" (p. 85). The proof uses the same principle: the contrary of the theorem would lead to an infinite product.

Both proofs are based on the principle that has been traditionally formulated as:

There is no infinite descending chain of natural numbers.

In the Lehrbegriff of 1797, this principle is made explicit in the proof of the prime divisor theorem: Schultz writes about the numbers in the sequence of divisors of a composite number that "they cannot become always smaller without end "(p. 56)

Overall we have: there is no trace of induction in the form of the step from $n$ to $n+1$ in the Anfangsgründe, but just one equational proof and two by the impossibility of an infinite sum and product; altogether unusual arguments. In 1797, the former has been changed into a canonical induction from $n$ to $n+1$, the latter into the well-known infinite descent argument. Did Schultz realize the equivalence of all these formulations? I don't think an answer would make such a big difference in the way we look at his results. We know they are based on one formulation or other of a principle today called induction. All four forms of induction found in Schultz have been used indiscriminately in arithmetic and elsewhere, with varying degrees of awareness of their equivalence.

\section{Recurrence in combinatorics}

The first explicit references to a "recurrent procedure" (rekurrierendes Verfahren) seem to stem from a development quite different from the foundational concerns of Schultz, namely from the combinatorics in the early 19th century. Andreas von Ettingshausen's (1796-1878) book Die combinatorische Analysis als Vorbereitungslehre zum Studium der theoretischen höhern Mathematik, published in 1826 , is one such (Combinatorial analysis as a preparatory for the study of theoretical higher mathematics). Combinatorial formulas are there described as "recurrent determinations" (recurrirende Bestimmungen), in the following general terms (p. 83):

... this procedure is the recurrent one, i.e., one in which a successive member of the result aimed at is obtained only when all preceding members have already been calculated.

The engaging book by Friedrich Wilhelm Spehr (1799-1833), in the second edition of 1840, has the exhaustive title Vollständiger Lehrbegriff der reinen Combinationslehre mit Anwendungen derselben auf Analysis und Wahrscheinlichkeitsrechnung (Complete course for learning the pure calculus of 
combinatorics with applications thereof to analysis and the calculus of probability). The preface and first edition are dated 1824 but considering its talented author's death at the young age of 34 seven years before the second edition, it has most likely been a simple reprint. In combinatorics, the nature of the things plays no role, but one considers just the ways in which they can be put together, writes Spehr (p. 1). Each topic is treated separately by the "independent" and the "recurrent" method, explained as follows (p. 9):

Whenever quantities or just things whatever are hand that are connected together successively according to laws that remain always the same, so that these connections are members of a progression, the members have also among themselves a fixed link; a rule can be given each time by which one can find another thing from some given ones. It is so overall in analysis where the lawful sequence comes out as a result of an operation, and it is so also in combinatorics.

One can produce each member of a result in itself and independent of every other member, from the quantities or things the connections of which shall bring it forth; (independent procedure, independent determination) but one can also derive a successive one from members of the result already formed earlier. (Recurrent procedure, recurrent determination.)

In $\S 15$, the recurrent method is explained in detail, with combinatorial formulas that show how the computation with a parameter value $k$ is reduced to one with a parameter value $k-1$. Such experience with complicated situations of combinatorics had led to the idea of a general pattern of reduction, in which in the end the value 1 is reached. Then, turning the procedure around, the value of an expression for $k$ could be determined step by step from the value for 1 .

\section{Grassmann's formalization of calculation}

In his Ausdehnungslehre of 1844 that introduced linear algebra and the vector calculus, Hermann Grassmann wrote (p. xix):

Proof in the formal sciences does not go into another sphere, beyond thinking itself, but resides purely in the combination of the different acts of thought. Therefore the formal sciences must not begin with axioms unlike the real ones; their foundation is made up of definitions. If axioms have been introduced into the formal sciences, such as arithmetic, this is to be seen as a misuse that can be explained only through the corresponding treatment in geometry.

The last quoted sentence is from an added footnote.

(A) Grassmann's discovery of definition by recursion. Fifteen years after the above passage was published, Grassmann put his credo about avoiding axioms in arithmetic to full effect through the application of the "recurrent procedure" to the most elementary parts of arithmetic, namely the basic arithmetic operations. His 1861 Lehrbuch der Arithmetik für höhere Lehranstalten contains the first explicit recursive definitions of arithmetic operations, ones that go hand in hand with inductive proofs of properties of the recursively defined operations. The base case of induction is 0 , or, in Grassmann's times, usually 1, and the step case is that of $n$ to $n+1$, the successor of $n$ [Nachfolger]. Grassmann's definition of sum is for an arbitrary "basic sequence" with a unit $e$ and a succession of units as in $e, e+e, e+e+e, \ldots$, with the addition of a unit always intended at right, in the sense of $(e+e)+e$. He actual! ly constructs a doubly infinite series of integers with a positive and negative unit, but the following is for brevity the part on natural numbers that begin with the positive unit denoted $e$. Here is Grassmann's explanation of the positive integers (p. 2):

7. Explanation. Let a sequence of quantities be built out of a quantity $e$ [Grösse] through the following procedure: One sets $e$ as one member of the sequence, $e+e$ (to be read $e$ plus $e$ ) as the successive [nächstfolgende] member of the sequence, and one continues in this way, by deriving from the member that is each time last the successive one through the joining of $+e$. 
When one assumes each member of this sequence to be different from all the other members of the sequence, one calls this sequence the basic sequence [Grundreihe], e the positive unit.

It is remarkable that Grassmann does not use the word "addition" in this characterization of the number sequence. The addition of the unit $e$ is explained as follows (p. 3):

8-9. Explanation. If $a$ is any member of the basic sequence, one means by $a+e \ldots$ the member of the sequence that follows next to $a \ldots$ that is, if $b$ is the member of the sequence next to $a$, we have

(8) $b=a+e$

One calls this operation [Verknüpfung] the addition of a unit.

Addition of a unit is a clearly defined separate operation explained as the taking of a successor in the basic series. Grassmann now sets out to prove that "the members of the sequence that follow $e$ are sums of positive units." The proof goes as follows:

Proof. The members of the basic series that follow $e$ have (by 7) resulted from $e$ by a progressive addition of positive units, are therefore sums of positive units.

There is a clear awareness in Grassmann that the sequence of natural numbers is generated from the unit through repeated application of the successor operation. The only fault is that there is no separate notation for the successor; it is denoted by $+e$. Grassmann's definition of addition should be read with the overloading of the symbol + in mind (p. 4):

15. Explanation. If $a$ and $b$ are arbitrary members of the basic sequence, one understands with the sum $a+b$ that member of the basic sequence for which the formula

$$
a+(b+e)=a+b+e
$$

holds.

In Grassmann's terms, this equation gives a procedure for reducing arbitrary sums into members of the "basic sequence" that by the above proof are "sums of positive units."

The recursive definition of sum is put into use in Grassmann's "inductory" (inductorisch) proofs of the basic properties of addition, such as associativity and commutativity. Anyone who tries to prove the commutativity of addition, $a+b=b+a$, will notice that the proof requires as a preliminary a proof of associativity, $(a+b)+c=a+(b+c)$. This must have happened to Grassmann who offers the following proof (p. 8):

22.

$$
a+(b+c)=a+b+c .
$$

"Instead of adding a sum one can add the summands step by step," or "instead of adding two quantities step by step, one can add their sums."

Proof (inductorily in relation to $c$ ). Assume formula 22 to hold for whatever value of $c$, then we have

$$
\begin{aligned}
a+[b+(c+e)] & =a+[b+c+e] & & \text { by 15). } \\
& =a+(b+c)+e & & \text { (by 15). } \\
& =a+b+c+e & & \text { (by assumption). } \\
& =a+b+(c+e) & & \text { (by } 15) .
\end{aligned}
$$

Therefore, if formula 22 holds for whatever value $c$, it holds even for the one following next, thereby for all values that follow.

The use of parentheses could have made the steps a bit clearer. Next, as result 23, Grassmann proves the commutativity of addition. 
Grassmann conceived of the natural numbers in a completely abstract way, as a special case of his "basic sequences," evidence the occurrence of the number symbols in his treatise: Zero appears early on in his system, the notation 0 for the sum of the positive and negative units (p. 3):

$$
e+-e=0
$$

Zero is not yet a natural number but belongs to any basic sequence. The symbols 1, 2, and 3 appear much later, after the treatment of sum and its properties has been completed in $\S 3$, in the section on Multiplication (\$ 4, p. 17):

52. Expl. By $a .1$ (read $a$ times one or $a$ multiplied by one) one intends the quantity $a$ itself, i.e.,

$$
\text { (52). } a .1=a \text {. }
$$

"To multiply by one changes nothing."

53. Expl. A basic sequence the unit of which is equal to one is called a number sequence, its members numbers, the number $1+1$ is denoted by 2 , the number $2+1$ by 3 , etc.

Grassmann ends this explanation by the remark that since the number sequence is a basic sequence, the previously established laws of addition and subtraction apply to it.

Multiplication with "the rest of the numbers (beyond 1)" is defined by the recursion equation $a \cdot(\beta+1)=a \beta+a$ where " $\beta$ is a positive number" (p. 18). Grassmann takes $a \cdot 0=0$ to be part of the definitional equations of a product. Much later, it became clear that it is instead derivable from the recursion equation and one of the Peano axioms.

Finally, a remark about Schultz' reduction of the proof of commutativity of multiplication to the special case $1 \cdot n=n \cdot 1$ and the distributive law: The first step in the proof as given above was $n \cdot 2=n \cdot(1+1)=n \cdot 1+n \cdot 1$, now an instance of Grassmann's recursive definition of product by the equation $a \cdot(b+1)=a \cdot b+a$, together with equation 52. Both equations are present in Schultz, but as "corollaries" instead of definitions.

Today, one would formulate the successor operation as a separately written function, with the notation $s(a)$ or $\operatorname{succ}(a)$ so as not to mix sum and successor, with 0 included and the definition of sum and product as:

$$
\begin{aligned}
& \text { 1. } a+0=a \quad a \cdot 1=a \\
& \text { 2. } a+s(b)=s(a+b) \quad a \cdot s(b)=a \cdot b+a
\end{aligned}
$$

Here it is seen how recursion clause 2 makes the second summand diminish step by step, until it becomes 0 and vanishes; thus, a clear conceptual advantage from having the natural numbers begin with 0 instead of 1 .

The apparent circularity of the definition of sum, signaled as a defect by Frege and others in the 19th century, is seen in the Grassmann-style recursion equation:

$$
a+(b+e)=(a+b)+e
$$

In Grassmann, the end of recursive return is signaled by the appearance of $a+e$. With clauses 1 and 2 , instead, recursion produces a well-founded sequence through the application of clause 2 , until the sum-operation is eliminated by clause 1 .

Grassmann's motivations for developing formal arithmetic are remarkably clearly stated in the introduction to his book. He writes that it "claims to be the first strictly scientific elaboration of the discipline" (p. v). However, Grassmann got rather frustrated at the lack of interest in his work, and turned into linguistics where he became one of the leading authorities in the Sanskrit language.

(B) The reception of Grassmann's idea. Grassmann's approach to the foundations of arithmetic is found explained in detail in the first of Hermann Hankel's two-volume treatise Vorlesungen über die complexen Zahlen und ihre Functionen of 1867. The full title of the first volume is Theorie der complexen Zahlensysteme insbesondere der gemeinen imaginären Zahlen und der Hamiltonschen Quaternionen nebst ihren geometrischen Darstellung (Theory of complex number systems expecially of the common imaginary numbers and of the Hamiltonian quaternions together with their geometrical representation). He begins with a discourse about the natural numbers (p. 1): 
What it means to think or pose an object 1 time, 2 times, 3 times ...cannot be defined because of the fundamental simplicity of the concept of posing. An absolute, entire number $1,2,3 \ldots$ expresses that an object has to be posed $1,2,3 \ldots$ times, and it means $1 e, 2 e, 3 e \ldots$, the result of repeated posing of $e$.

Next addition is explained as the result of posing the numerical unit $e$ first $a$ times, then $b$ times. Associativity and commutativity are mentioned as "the main laws." Further, addition is an operation with a unique result and the property that if one summand is changed and the other remains constant, also the result changes (p. 2):

The properties of addition given here are sufficient for the derivation of all further consequences on the building of sums, without the need to remind oneself each time about the real meaning of addition. In this sense they are the conditions that are necessary and sufficient to formally define the operation.

"Positive entire numbers" are the topic of $\S 9$, generated from the unit 1 by setting $1+1=2,2+1=$ $3,3+1=4, \ldots$ The sum $(A+B)$ of two numbers is defined as in Grassmann's recursion equation (p. 37):

$$
A+(B+1)=(A+B)+1 .
$$

Hankel now states that "this equation determines every sum" and shows how it goes: By setting $B=1$ in the equation one has $A+2=A+(1+1)=(A+1)+1$, and with $B=2$ one has $A+3=A+(2+1)=(A+2)+1$ so that $A+2$ and $A+3$ are numbers in the sequence of integers (p. 37):

In this way one finds through a recurrent procedure, one that goes on purely mechanically without any intuition, unequivocally every sum of two numbers ${ }^{1}$

This proclamation is followed by a mechanical computation of the Kantian formula $7+5=12$ through the writing of 17 equations that begins with $7+5=7+(4+1)=(7+4)+1$ and ends with $7+5=$ $11+1=12$. The example is very likely inspired by the proof in Schultz' Prüfung as discussed above. Next Hankel proves associativity, then the lemma $1+A=A+1$, and finally commutativity exactly as in Grassmann, and proceeds then to the recursive definition of multiplication. The presentation of positive integers ends with (p. 40):

The idea to derive the rules of addition and multiplication as done here owes itself in its essence to Grassmann (Lehrb. d. Arithmetik).

Grassmann's approach is next described by Ernst Schröder in his 1873 Lehrbuch der Arithmetik und Algebra. The book contains an introduction and chapters on arithmetic operations with a presentation that follows directly Hankel's divisions. The integers are explained through the process of counting, with the paradigm that "a natural number is a sum of ones" (p. 5). The addition of one is kept strictly separate from the concept of an arbitrary sum; the symbol + is used only because otherwise, writing for example 111 for three, one would take that as a hundred and eleven. A number is further "independent of the order in which the units are built with the unit stroke" (p. 16).

Each of the arithmetic operations is treated twice, just as in the much earlier book of combinatorics by Spehr: first by the independent treatment (independente Behandlungsweise), then by the recurrent one (p. 51). What the former is does not become clearly explained, but it is more or less the way one learns basic arithmetic at school, by becoming acquainted with counting and numbers and sums and so on. Spehr's explanation was that a solution can be determined in itself for a given case, independent of other possible cases. The second approach aims at greater rigor and "a simplification of the conditions taken as a point of departure." Grassmann's recurrent mode of counting sums is explained through detailed examples (pp. 63-64):

\footnotetext{
1 The German, set in fraktur, is too beautiful to be left just in translation: Auf diese Weise findet man durch ein recurrirendes Verfahren, welches ohne alle Anschauung, rein mechanisch vor sich geht, unzweideutig jede Summe zweier Zahlen.
} 
(5) $2=1+1,3=2+1,4=3+1,5=4+1$, etc.,

The natural numbers are hereby defined recurrently. Namely, to give in a complete way the meaning of a number, i.e., to express it through the unity, one has to go back from it to the previous number and to run through backwards (recurrere) the whole sequence.

The presentation is copied from Hankel, up to the 17 equations that lead from $7+5=7+(4+1)$ to $7+5=11+1=12($ p. 65$)$ :

One can find such a sum [of two numbers] in this way unequivocally, through a recurrent procedure that goes on purely mechanically.

Well, this goes over to the side of direct copying of Hankel. In counterbalance, Schröder's presentation of how the natural numbers are generated by the +1 -operation is described through a notational novelty (p. 64):

If $a$ is a number from our sequence: $1,2,3,4,5, \ldots$ then even $a+1$ is one, namely

$$
a^{\prime}=a+1
$$

is the general form of equations (5).

This seems to be the first place in which the successor operation obtains a separate notation, one that became later the standard one. It was a conceptually important step and the notation was taken into use by Dedekind in 1888 .

As a conclusion, we can say that with the two textbooks by Hankel and Schröder, Grassmann's recursive foundation of arithmetic became known and generally appreciated. This is made evident through Richard Dedekind's widely read booklet Was sind und was sollen die Zahlen? of 1888 (What are numbers and what are they for?). On the first page, Dedekind mentions as the first source Schröder's "exemplary Lehrbuch"; one finds then in Dedekind the use of the successor function as a primitive, with the Schröderian notation $p=n^{\prime}$ for "the successive number" (p. 27). Addition is defined by the recursive clauses (p. 36):

$$
\begin{aligned}
& \text { II. } m+1=m^{\prime} \\
& \text { III. } m+n^{\prime}=(m+n)^{\prime}
\end{aligned}
$$

Next the basic properties are proved inductively, with a slight variant of the old order of associativity of sum followed by its commutativity: Dedekind proves first $m^{\prime}+n=m+n^{\prime}$ (Satz 136), from clauses II and III silently using even the substitution principle in the successor function, from $m=n$ to conclude $m^{\prime}=n^{\prime}$, with the intermediate step $m^{\prime}+n=(m+n)^{\prime}$ that with clause III gives the result (p. 36). Now follow proofs of $1+n=n+1$ and $m+n=n+m$ that don't use 136, and then the standard proof of associativity expressed as $(l+m)+n=l+(m+n)$. The order of things is somewhat redundant: Associativity with the instance $(m+1)+n=m+(1+n)$ together with $1+n=n+1$ gives at once Dedekind's Satz 136. His intermediate step $m^{\prime}+n=(m+n)^{\prime}$ in the proof is the more interesting result because it shows that the recursion clause can be applied as well to the first argument of a sum.

\section{Peano: the logic of Grassmann's formal proofs}

(A) The Peano axioms. Giuseppe Peano published in 1889 a separate little treatise, the 36-page Arithmetices Principia, Nova Methodo Exposita, or "The principles of arithmetic, presented by a new method." It was written in Latin and the earlier parts got an English translation in the Van Heijenoort collection in 1967, From Frege to Gödel. The original is readily available on-line and one sees that this booklet consists of a 16-page preface and explanation, and a 20-page systematic development that begins with $\S 1$ : On numbers and on addition. Peano writes in the introduction (Van Heijenoort's translation, p. 85): 
I have denoted by signs all ideas that occur in the principles of arithmetic, so that every proposition is stated only by means of these signs.

With these notations, every proposition assumes the form and the precision that equations have in algebra; from the propositions thus written other propositions are deduced, and in fact by procedures that are similar to those used in solving equations.

Peano uses dots in place of parentheses, $a \cap b$ and $a b$ for conjunction, $-a$ for negation, $V$ for truth, and the same inverted for falsity $\Lambda$. Implication is written $a \supset b$, read "deducitur" (one deduces), but it is clearly a connective, because it is found iterated. For example, Peano's second propositional axiom is:

$a \supset b . b \supset c: \supset . a \supset c$

There is also the connective of equivalence, $a=b$, definable through implication and conjunction as $a \supset b . \cap . b \supset a$.

Peano writes in the preface that he has followed in logic amongst others Boole, and for proofs in arithmetic the book by Grassmann (1861) [in arithmeticae demonstrationibus usum sum libro: H. Grassmann]. Among his references there is also the book of Schröder of 1873. There is no evidence of the influence of Frege, even if some of the initial statements about the ambiguity of language and the necessity to write propositions only in signs are very close to those in Frege's Begriffsschrift. Dedekind (1888) is mentioned as a "a useful recent book" in which "questions pertaining to the foundations of numbers are acutely examined."

Definitions are explained as follows (p. 93):

A definition, or Def. for short, is a proposition of the form $x=a$ or $\alpha \supset . x=a$, where $a$ is an aggregate of signs having a known sense, $x$ is a sign or aggregate of signs, hitherto without sense, and $\alpha$ is the condition under which the definition is given.

Pure logic is followed by a chapter on classes, or sets as one could say. The notation is $a \varepsilon b$ for $a$ is $a b$, and $a \varepsilon K$ for $a$ is a class.

To get to arithmetic, Peano adds to the language the symbols $N$ (number), 1 (unity), $a+1$ (a plus 1 ), and = (is equal to), the same symbol is used also for logic. The Peano axioms for the class $N$ of natural numbers are:

\section{Peano's axioms for natural numbers:}

1. $1 \varepsilon N$

2. $a \varepsilon N . \supset . a=a$

3. $a, b \varepsilon N . \supset: a=b .=. b=a$

4. $a, b, c, \varepsilon N . \supset \therefore a=b . b=c: \supset . a=c$.

5. $a=b . b \varepsilon N: \supset . a \varepsilon N$.

6. $a \varepsilon N . \supset . a+1 \varepsilon N$.

7. $a, b \varepsilon N . \supset: a=b .=. a+1=b+1$.

8. $a \varepsilon N . \supset \cdot a+1-=1$.

9. $k \varepsilon K \therefore 1 \varepsilon K \therefore x \varepsilon N . x \varepsilon k: \supset_{x} \cdot x+1 \varepsilon k:: \supset . N \supset k$.

In axioms 2,7 , and 8 with its negated equality, equality of numbers and equivalence of propositions get happily mixed.

One direction of axiom 7 can be seen as a principle of replacement of equals $a=b$ in the successor function $a+1$. The remaining direction together with axiom 8 are often referred to as "the axioms of infinity," after Dedekind (1888): They show that the positive natural numbers $N^{+}$, a proper subset of $N$, are in a one-to one correspondence with $N$, which makes $N$ infinite in Dedekind's definition.

The last axiom is the principle of induction: Let $k$ be a class that contains 1 and for any $x$, let it contain $x+1$ if it contains $x$. Then it contains the class $N$. The implication has the eigenvariable $x$ of the inductive step as a subscript. 
After the axioms there is a definition of sorts:

10. $2=1+1 ; 3=2+1,4=3+1$; and so forth.

Peano's definition, or rather "definition," contains the same defect as those of Grassmann, Hankel, and Schröder, revealed by the elliptic "etc" or similar, namely that no way is given for inductively producing arbitrary decimal expressions from expressions in pure successor form.

There follows a great number of theorems the proofs of which consist of two kinds of steps, with $b$ a substitution instance of axiom $a$ in the first:

$$
\begin{aligned}
& a \supset b . \\
& a . a \supset b: \supset b .
\end{aligned}
$$

From the derivations in Peano's treatise, the following structure emerges, as shown in detail in von Plato (2013, section 14.3(b)): Peano's formal derivations consist of a succession of formulas that are either implications with an axiom that implies its instance, or implications with two derived formulas $a$ and $a \supset b$ that imply $b$.

Jean van Heijenoort who edited the book that contains the first English translation of the main part of Peano's 1889 work, instead of figuring out what Peano's notation for derivations means, claims in his introduction (p. 84) that there is "a grave defect. The formulas are simply listed, not derived; and they could not be derived, because no rules of inference are given... he does not have any rule that would play the role of the rule of detachment." Little is needed to see that Peano's derivations are instead constructed purely formally, with a notation as explicit as one can desire, by the application of axiom instances and implication eliminations.

After the above theorem numbered 11 in Peano, there follow other very simple consequences about the equality relation, numbered $12-17$. Next comes the definition:

18.

$$
a, b \varepsilon N . \supset . a+(b+1)=(a+b)+1 .
$$

Peano notes (p. 95):

Note. This definition has to be read as follows: if $a$ and $b$ are numbers, and if $(a+b)+1$ has a sense (that is, if $a+b$ is a number) but $a+(b+1)$ has not yet been defined, then $a+(b+1)$ signifies the number that follows $a+b$.

Peano gives as examples of the use of the definition formal computations of the values $a+2$ and $a+3$, written as:

$$
\begin{aligned}
& a+2=a+(1+1)=(a+1)+1 \\
& a+3=a+(2+1)=(a+2)+1
\end{aligned}
$$

Combining these two, we get:

$$
a+3=a+(2+1)=(a+2)+1=(a+(1+1)+1=((a+1)+1)+1
$$

Now begins a section with theorems, clearly ones suggested by those in Grassmann, and with inductive proofs. Number 19 shows that natural numbers are closed with respect to addition, number 22 is a principle of replacement of equals in a sum, by which $a=b \supset a+c=b+c$, and 23 is the associative law. To arrive at commutativity, Peano proves first as 24 the lemma $1+a=a+1$, then finishes with 25 that is commutativity of addition to 28 that is replacement at both arguments in sums, $a=b . c=d \supset a+c=b+d$. The part on natural numbers is finished by sections on the recursive definition and basic properties of subtraction, multiplication, exponentiation, and division, all of it following Grassmann's order of things in definitions and theorems to be proved ( $\$ 2-6)$.

Peano adds the consideration of classes to Grassmann, and therefore he has operations such as $M$ for "the maximum among...," and the same inverted for minimum. Thus, he expresses Euclid's result about the infinity of primes numbers as result 23 in $\S 3$, with $N p$ standing for the class of prime numbers:

23.

$$
M \cdot N p:=\Lambda
$$


Here $\Lambda$ is the sign of absurdity or the empty class. The whole is read as something like "the maximum among prime numbers is equal to an empty class."

Van Heijenoort's comments on recursive definition in Peano are also flawed, though not as terribly as those about deduction. He writes about Peano's definition of addition and multiplication (p. 83):

Peano ... puts them under the heading "Definitions", although they do not satisfy his own statement on that score, namely, that the right side of a definitional equation is "an aggregate of signs having a known meaning".

When introducing his primitive signs for arithmetic, Peano enlisted unity, notation 1, and a plus 1 , notation $a+1$. Thus, the sum of two numbers was not a basic notion, but just the successor, and definition 18 laid down what the addition of a successor $b+1$ to another number means, in terms of his primitive notions. Peano explained the matter carefully in the note after definition 18, cited above. If, as Peano assumes, $a+b$ is a number, i.e., if $a+b \varepsilon N$, then even $(a+b)+1 \varepsilon N$, so the definiens "has a meaning" as Peano writes, and one really wonders what Van Heijenoort may have been thinking here, if anything. Peano's misfortune was perhaps to use the same notation for the operation of a successor and for an arbitrary sum.

The notation for natural numbers and their operations got clearly improved in the project "Formulario mathematico," a series of five versions of a book in which mathematics was to be developed within Peano's formalism. The title amounts to something like "Formula collection for mathematics", published in four French versions between 1895 and 1903 with the title Formulaire de Mathématiques and Formulaire Mathématique, and the fifth and last in Peano's own artificial language latino sine flessione as the Formulario Mathematico (1908). (N.B. one might wonder what else "Latin without inflexions" could be than Italian slightly contorted.)

In the Formulario of 1901, there is a clear stylized implication symbol, identical to one used here, and also the existential quantifier, an inverted upper case E, but just the axiom by which an instance gives existence (p. 28). The natural numbers begin with 0 , and the successor is written as $a+$, "the number that comes after $a$, the successor of $a, a$ plus," as if the second summand had been left blank (p. 39). The recursion equations for sum are (p. 40):

$$
\begin{array}{ll}
\cdot 1 & a \in N_{0} \cdot \supset \cdot a+0=a \\
\cdot 2 & a, b \in N_{0} \cdot \supset \cdot a+(b+)=(a+b)+
\end{array}
$$

The definition $1=0+$ gives now $a+1=a+(0+)=(a+0)+=a+$ and $a+(b+1)=(a+b)+1$.

\section{Skolem's recursive arithmetic}

Thoralf Skolem (1887-1963) became aware of the Frege-Russell tradition of logic only in 1919; his reaction to Russell's universal and existential quantifiers was one of rejection, with an alternative quantifier-free theory of arithmetic as based on recursive definitions. The work got published only in 1923 in a rather obscure place, having been written in the Fall of 1919 but rejected by the prestigious journal Acta Mathematica. Nevertheless, it found its readers, Paul Bernays in particular.

Skolem's motivation for developing quantifier-free arithmetic comes out when defines the notion of divisibility $D(a, b)$, with $\Sigma_{x}$ the Schröderian notation for the existential quantifier (p. 160):

$$
D(a, b) \equiv \Sigma_{x}(a=b x)
$$

The crucial observation is:

Such a definition refers to an infinite - and that is to say unexecutable - work, for the criterion of divisibility consists in trying out, the whole series of numbers through, whether one can find a number $x$ such that $a=b x$ obtains.

Skolem notes at once that the definition can be given by the use of bounded quantifiers: (p. 161):

$$
D(a, b) \equiv \sum_{1}^{a} x(a=b x) \equiv((a=b)+(a=2 b)+(a=3 b)+\cdots+(a=b a)) .
$$


The bound variable $x$ has a finite upper bound, and Skolem concludes:

Therefore this definition gives us a finite criterion of divisibility: one can determine in each case through a finite work - a finite number of operations - whether the proposition $D(a, b)$ holds or not.

Skolem's dissatisfaction with Russell's quantifiers was not just that: In Frege and Russell, there is a totally failed attempt at founding the basic concepts of arithmetic on a heavy logical apparatus. Arithmetic based on recursive definitions, absent from Frege's and Russell's involved theories that don't lead anywhere, is the right way for Skolem. In a paper titled The development of recursive arithmetic of 1947 Skolem writes about his primitive recursive arithmetic (p. 500):

\begin{abstract}
After having studied the Principia Mathematica (Russell-Whitehead 1910-13) in 1919 I discovered that a great part of ordinary number theory could be developed without quantifiers... A paper containing my considerations was published in 1923, and is so far as I know the first investigation in recursive number theory. The utterance of H. B. Curry (1941) that the recursive arithmetic can be traced back to Dedekind and Peano seems to me rather strange, because the researches of these men had another purpose than to avoid the use of quantifiers.
\end{abstract}

This passage contains an oddity, namely, Dedekind and Peano had no theory of quantifiers at their disposal around 1890, even less to avoid, as that became accessible only through the very same Principia to which Skolem refers. Skolem is clearly upset by Curry's remark, a brief one (1941, p. 263): "Such a treatment of arithmetic was initiated by Skolem -although the essential ideas were already in Dedekind and Peano."

A later paper of Skolem's, The logical background of arithmetic of 1955, contains what Skolem takes to be "the most important views concerning the logical nature of arithmetic which were set forth in the latter half of the 19th century" (p. 541). The only 19th century figure mentioned is Dedekind. However, a reading of Skolem's 1923 paper, in comparison to the treatments of Grassmann, Peano, and Schröder, reveals similarities that are too striking to be casual coincidences. It is good to keep in mind that all of Skolem's early work was based on Schröder's Vorlesungen über die Algebra der Logik. The beginnings of Schröder's approach are already seen in the earlier Lehrbuch der Arithmetik und Algebra, alongside its clear exposition of Grassmann's recursive arithmetic. Finally, Peano's 1889 presentation of arithmetic follows strictly Grassmann so that Peano would have been a possible source for Skolem, despite his belittlement of Peano's present! ation of recursive arithmetic. Skolem's order of things is indeed almost identical to Peano's, with just subtraction treated after order and product, as in Schröder's presentation. The last-mentioned has many more topics because of the inclusion of the "independent treatment." Dedekind, whose little treatise Was sind und was sollen die Zahlen? of 1888 was certainly known to Skolem, has on the very first side the following:

Of the literature known to me I mention the exemplary Lehrbuch der Arithmetik und Algebra of Ernst Schröder's (Leipzig 1873) in which one finds even a list of the literature.

The mathematics library of the University of Oslo has had a copy of Schröder's 1873 book in addition to the extensive three-volume work on algebraic logic, the former lost not very long ago as I found out during a visit in May 2015. Skolem had put many years into the study and development of Schröder's algebraic logic. It would be very strange if as a student in Oslo he had not at least glanced through a praised book on arithmetic by the same author, then perhaps to forget where the basic ideas of recursive arithmetic came from.

\title{
7 Computation turned into deduction
}

The close to hundred-page chapter 7 on recursive arithmetic in the Grundlagen der Mathematik was the first systematic presentation of the topic. It owes a lot to Skolem's 1923 paper, but Bernays 
refers even to Peano's Formulario in the last 1908 edition, to Peano's original work of 1889, and to Dedekind (1888).

There is a wonderful, clear insight in the beginning pages of the chapter, namely that the recursive definition of basic arithmetic operations turns computation into deduction, repeated several times. The first one was quoted above in the introductory section. Here are another two (p. 290, p. 292):

If $c$ is a numeral, the computation of the value of $f(a, \ldots, t, x)$ is already completely formalized through the derivation of the equation

$$
f(a, \ldots, t, x)=c
$$

We can reconstruct completely the recursive procedure of computation of finitary number theory in our formalism, through the deductive application of the recursion equations.

In $\S 6$, Bernays had given a consistency proof for a weak system of arithmetic that contains no recursive definitions. An example is given that shows how the consistency result can go lost under the addition of recursive definitions, with the more general conclusion (p. 301)

The situation found here can be set straight also from the intuitive point of view. A system of two equations of the form

$$
\begin{aligned}
& f(0)=a, \\
& f\left(n^{\prime}\right)=b(n, f(n))
\end{aligned}
$$

puts a requirement on the function $f(n)$. Whether it is satisfiable is not revealed by the structure of the recursion equations themselves, but depends instead on the characteristic properties of the successor function, namely that this function never leads to the value 0 , and that two different argument values always correspond to two different values of the successor function.

Therefore the admission of recursive definitions amounts to an implicit characterization of the successor function.

The insight leads Bernays to assume that Peano's two infinity axioms can be derived by recursive definitions (pp. 302-303). The first infinity axiom $a^{\prime} \neq 0$ can indeed be derived from $0^{\prime} \neq 0$ and the function Bernays designates by $\alpha$ (p. 302):

$$
\begin{aligned}
& \alpha(0)=0 \\
& \alpha\left(n^{\prime}\right)=0^{\prime}
\end{aligned}
$$

The equality axiom gives $a^{\prime}=0 \Rightarrow \alpha\left(a^{\prime}\right)=\alpha(0)$, then the recursion equations $0^{\prime}=0$ against the axiom $0^{\prime} \neq 0$, so that $a^{\prime} \neq 0$ follows.

The second infinity axiom $a^{\prime}=b^{\prime} \Rightarrow a=b$ is derived by the predecessor function $\delta$ in the now standard way (p. 302):

$$
\begin{aligned}
& \delta(0)=0 \\
& \delta\left(n^{\prime}\right)=n
\end{aligned}
$$

The equality axiom gives again $a^{\prime}=b^{\prime} \Rightarrow \delta\left(a^{\prime}\right)=\delta\left(b^{\prime}\right)$, and the second recursion equation then $a=b$.

It is worth while to compare Bernays' interpretation to that of Dedekind. In the latter, the first infinity axiom shows that the image of $N$ under the successor function is a proper subset of $N$, the second axiom gives a one-to-one correspondence between this subset and and $N$, so Dedekind's two clauses for the infinity of a set are satisfied. In Bernays, instead, the second axiom guarantees that when in a recursive call the step back from a successor $n^{\prime}$ is taken, the new argument $n$ of the recursive function is unique. Similarly, the first axiom just tells that recursive call ends when 0 is reached.

The appearance of the first volume of the Grundlagen made formal computation a clearly defined topic of its own. Progress in the theory of computability was remarkable fast, with several equivalent 
definitions of the notion of a recursive function, Church's thesis, and Turing's fundamental result about the undecidability of the halting problem given by 1936 .

Note added in proof: A referee has pointed out that the recursive methods in combinatorics of Section 3 above can be traced back to the work of C. F. Hindenburg from the 1790s on.

\section{References:}

Curry, H. (1941) A formalization of recursive arithmetic. American Journal of Mathematics, vol. 63, pp. 263-282.

Dedekind, R. (1888) Was sind und was sollen die Zahlen?

von Ettingshausen, A. (1826) Die combinatorische Analysis als Vorbereitungslehre zum Studium der theoretischen höhern Mathematik. Wallishauser, Vienna.

Grassmann, H (1844) Die Wissenschaft der extensiven Grösse oder die Ausdehnungslehre. Wigand, Leipzig.

Grassmann, H (1861) Lehrbuch der Arithmetik für höhere Lehranstalten. Enslin, Berlin.

Hankel, H. (1867) Vorlesungen über die complexen Zahlen und ihre Functionen I. Voss, Leibzig.

van Heijenoort, J., ed, (1967) From Frege to Gödel, A Source Book in Mathematical Logic, 1879-1931.

Harvard University Press.

Hilbert, D. and P. Bernays $(1934,1939)$ Grundlagen der Mathematik I-II. Springer.

Martin, G. (1938) Arithmetik und Kombinatorik bei Kant. References to the second edition, de Gruyter, Berlin 1972.

Peano, G. (1889) Arithmetices Principia, Nova Methodo Exposita. Partial English tr. in Van Heijenoort.

Peano, G. (1901) Formulaire de Mathématiques. Carré et Naud, Paris.

von Plato, J. (2007) In the shadows of the Löwenheim-Skolem theorem: early combinatorial analyses of mathematical proofs. The Bulletin of Symbolic Logic, vol. 13, pp. 189-225.

von Plato, J. (2013) Elements of Logical Reasoning. Cambridge.

von Plato, J. (2014) Generality and existence: quantificational logic in historical perspective. The Bulletin of Symbolic Logic, vol. 20 (2014), pp. 417-448.

Schultz, J. (1789, 1992) Prüfung der Kantischen Critik der reinen Vernunft I-II. Hartung, Königsberg.

Schultz, J. (1790) Anfangsgründe der reinen Mathesis. Hartung, Königsberg.

Schultz, J. (1797) Kurzer Lehrbegiff der Mathematik. Nicolovius, Königsberg.

Schröder, E. (1873) Lehrbuch der Arithmetik und Algebra für Lehrer und Studirende. Erster band. Die Sieben algebraischen Operationen. Teubner, Leipzig.

Skolem, T. (1919) Untersuchungen über die Axiome des Klassenkalküls und über Produktations- und Summationsprobleme, welche gewisse Klassen von Aussagen betreffen. As reprinted in Skolem 1970, pp. 67-101.

Skolem, T. (1923) Begrüngung der elementaren Arithmetik durch die rekurrierende Denkweise ohne Anwendung scheinbarer Veränderliche mit unendlichem Ausdehnungsbereich. As reprinted in Skolem 1970, pp. 153-188. English tr. in Van Heijenoort.

Skolem, T. (1930) Über einige Satzfunktionen in der Arithmetik. As reprinted in Skolem 1970, pp. 281306.

Skolem, T. (1947) The development of recursive arithmetic. As reprinted in Skolem 1970, pp. 499-514.

Skolem, T. (1955) The logical background of arithmetic. As reprinted in Skolem 1970, pp. 541-552.

Skolem, T. (1970) Selected Works in Logic. ed. J. E. Fenstad, Universitetsforlaget, Oslo.

Spehr, F. (1840) Vollständiger Lehrbegriff der reinen Combinationslehre mit Anwendungen derselben auf Analysis und Wahrscheinlichkeitsrechnung. Second edition, Leibrock, Braunschweig.

Waismann, F. (1936) Einführung in das mathematische Denken. Cited from the English tr. Introduction to Mathematical Thinking, Harper, several eds.

Whitehead, A. and B. Russell (1910-13) Principia Mathematica. Vols. I-III. 